\title{
Optimal Relay UAV Guidance-A New Differential Game ${ }^{1}$
}

\author{
Youngdong Choi ${ }^{2}$, Meir Pachter ${ }^{3}$, David Jacques ${ }^{4}$ \\ Air Force Institute of Technology \\ Wright-Patterson AFB, OH 45433
}

\begin{abstract}
A new differential game which admits a "closed form" solution is analyzed. In this paper guidance laws are developed to optimally position a relay Micro-UAV (MAV) to provide an operator at the base with real-time Intelligence, Surveillance, and Reconnaissance (ISR) by relaying communication and video signals when the rover MAV performing the ISR mission is out of radio contact range with the base. The ISR system is comprised of two MAVs, the Relay and the Rover, and a Base. The Relay strives to minimize the radio frequency (RF) power required for maintaining communications, while the Rover performs the ISR mission, which may maximize the required $R F$ power. The optimal control of the Relay MAV entails the solution of a differential game.
\end{abstract}

\section{INTRODUCTION}

$\mathrm{U}$ nmanned Aerial Vehicles (UAVs) are prevalent in current military operations. UAVs vary in size and mission. While some UAVs are the same size as aircraft, others are man-portable and can be carried in a backpack. These man-portable Micro-UAVs (MAVs) utilized by small tactical units are not supported by satellite communications and use radio frequency (RF) modems. High frequency radio communications are range limited. The MAVs considered in this paper are utilized for Intelligence, Surveillance and Reconnaissance (ISR) and will therefore be referred to as ISR MAVs or as Rovers [1]. The Base may lose communication (and controllability) with deployed ISR MAVs/Rovers if the Rovers, in the course of performing their mission, stray far away. In this paper guidance laws are developed to optimally position an autonomous Relay MAV to provide the operator at the Base with real-time ISR by relaying communication and sensor data while allowing for extended range Rover operations. The Relay-Rover interaction is modeled as a differential game whose solution yields the optimal Relay strategy.

\section{ANALYSIS}

It is assumed that the rElay (E) MAV is cognizant of the rOver's (O) instantaneous position and, obviously, own ship position. As far as the RF power requirements are concerned, this is determined by their distance from the Base

\footnotetext{
${ }^{1}$ The views expressed in this paper are those of the authors and do not reflect the official policy or position of the United States Air Force, Department of Defense, or the United States Government.

${ }^{2}$ Capt., R.O.K. Air Force, ychoi@afit.edu

${ }_{3}^{3}$ Professor, meir.pachter@afit.edu

${ }^{4}$ Associate Professor, david.jacques@afit.edu
}

(B) and the Rover-Relay separation. Thus, the state is the distance $r_{E}$ of the Relay from the Base, the distance $r_{O}$ of the Rover to the Base, and the angle $\theta$ included between the radials from the Base to the Relay and the Rover. This angle is measured clockwise. The MAVs have simple motion. The control for each MAV is its relative heading angle measured clock-wise from its radial from the Base. Figure 1 provides a visualization of the kinematics. The differential equations of motion are

$$
\left.\begin{array}{rlrl}
\dot{r}_{E}=V_{E} \cos \varphi & , r_{E}(0) & =r_{E_{0}} \\
\dot{r}_{O}=V_{O} \cos \psi & , r_{O}(0) & =r_{O_{0}} \\
\dot{\theta}=\frac{1}{r_{O}} V_{O} \sin \psi-\frac{1}{r_{E}} V_{E} \sin \varphi & , \theta(0) & =\theta_{0}, 0 \leq t \leq T
\end{array}\right\} .
$$

$T$ is the planning horizon utilized by the control algorithm.

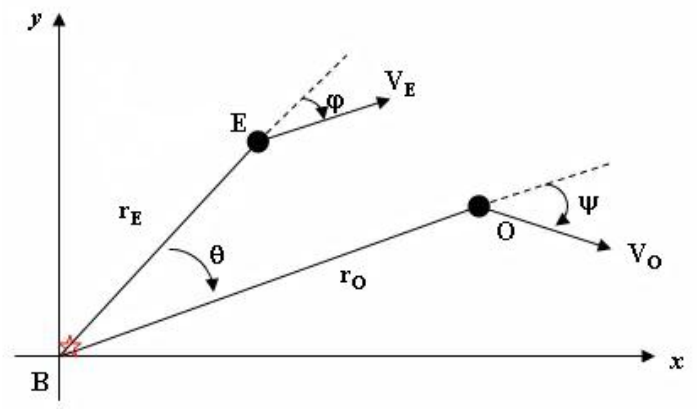

Fig. 1. Schematic of Relay System

The cost functional is indicative of the RF power required and is the time averaged sum of the squares of the distance between the Relay and the Rover and between the Relay and the Base:

$$
y=\int_{0}^{T}\left(\overline{E O}^{2}(t)+\overline{B E}^{2}(t)\right) d t
$$

The points $E, B$ and $O$ in $\mathbb{R}^{2}$ represent the positions of the Relay, Base and Rover respectively. These three points form a triangle which can be utilized to calculate the distance $\overline{E O}$ by the law of cosines.

$$
\overline{E O}^{2}(t)=r_{E}^{2}+r_{O}^{2}-2 r_{E} r_{O} \cos \theta
$$

Hence the cost functional is

$$
y=\int_{0}^{T}\left(2 r_{E}^{2}+r_{O}^{2}-2 r_{E} r_{O} \cos \theta\right) d t
$$

The objective is to minimize the average RF power required for maintaining communications. The control available to accomplish this task is limited to setting the course angle $\varphi$ of the Relay, while the Rover performs his 
ISR mission: in a worst case scenario, one might assume that the independently controlled Rover is working to maximize the cost functional. The optimization problem is then a differential game [2] where the Relay's control is its relative heading $\varphi$ and the Rover's control is its relative heading $\psi$.

The system is analyzed by first non-dimensionalizing the states and the parameters. The velocities are scaled by the velocity of the Relay $\left(V_{E}\right)$, yielding a non-dimensional speed ratio $\alpha$. The distances are scaled by $\mathrm{d}$, where $\mathrm{d}$ is a characteristic length, say $d=V_{E} T$. Set

$$
r_{E}:=\frac{r_{E}}{d}, r_{O}:=\frac{r_{O}}{d}, \mathrm{t}:=\frac{V_{E}}{d} \cdot t, \mathrm{~T}:=\frac{V_{E}}{d} \cdot T \text { and the speed }
$$

ratio $\alpha \equiv \frac{V_{O}}{V_{E}}$. Using these non-dimensional variables and parameters, the two sided optimization problem now becomes

$$
\begin{array}{rlrl}
\min _{\varphi} \max _{\psi} \mathcal{y} & =\int_{0}^{T}\left(2 r_{E}^{2}+r_{O}^{2}-2 r_{E} r_{O} \cos \theta\right) d t & \\
\text { s.t. } & & , r_{E}(0)=r_{E_{0}} \\
\dot{r}_{E} & =\cos \varphi \quad, r_{O}(0)=r_{O_{O}} \\
\dot{r}_{O} & =\alpha \cos \psi & & \\
\dot{\theta} & =\frac{1}{r_{O}} \alpha \sin \psi-\frac{1}{r_{E}} \sin \varphi, \theta(0) & =\theta_{0}, 0 \leq t \leq T
\end{array}
$$

The problem parameters are the speed ratio $\alpha \geq 0$ and the planning horizon $T>0$.

Since the optimal control problem only makes sense if the rOver is closer to the rElay than to the Base (B), the following must hold.

$$
r_{E} \leq 2 r_{O} \cos \theta
$$

Thus, since the problem is symmetric about the $\theta=0$ axis, the state space is

$$
\left\{\left(r_{E}, r_{O}, \theta\right) \mid 0 \leq \theta \leq \frac{\pi}{2}, 0 \leq r_{E} \leq 2 r_{O} \cos \theta\right\}
$$

To solve the differential game, the Hamiltonian is introduced in eq. (4),

$$
\begin{aligned}
\mathscr{H}= & -2 r_{E}^{2}-r_{O}^{2}+2 r_{E} r_{O} \cos \theta+\lambda_{r_{E}} \cos \varphi \\
& +\lambda_{r_{O}} \alpha \cos \psi+\lambda_{\theta}\left(\frac{1}{r_{O}} \alpha \sin \psi-\frac{1}{r_{E}} \sin \varphi\right)
\end{aligned}
$$

where $\lambda_{r_{E}}, \lambda_{r_{O}}$ and $\lambda_{\theta}$ are the system co-states.

According to the Pontryagin Maximum Principle (PMP) [3], the differential equations for the co-states are

$$
\begin{array}{ll}
\dot{\lambda}_{r_{E}}=4 r_{E}-2 r_{O} \cos \theta-\frac{\lambda_{\theta} \sin \varphi}{r_{E}^{2}}, \lambda_{r_{E}}(T)=0 \\
\dot{\lambda}_{r_{O}}=2 r_{O}-2 r_{E} \cos \theta+\frac{\lambda_{\theta} \alpha \sin \psi}{r_{O}^{2}}, \lambda_{r_{O}}(T)=0 \\
\dot{\lambda}_{\theta}=2 r_{E} r_{O} \sin \theta \quad, \lambda_{\theta}(T)=0
\end{array}
$$

and the optimality condition is given by $\max _{\varphi} \min _{\psi} \mathscr{H}$, namely the derivatives of $\mathscr{H}$ in $\varphi$ and $\psi$ vanish.

This yields

$$
\tan \varphi^{*}=-\frac{\lambda_{\theta}}{\lambda_{r_{E}} r_{E}}
$$

and

$$
\tan \psi^{*}=\frac{\lambda_{\theta}}{\lambda_{r_{O}} r_{O}}
$$

The second-order sufficiency condition for $\varphi$ is

$$
\therefore \frac{\partial^{2} \mathscr{H}}{\partial \varphi^{2}}=-\lambda_{r_{E}} \cos \varphi+\frac{\lambda_{\theta} \sin \varphi}{r_{E}}<0
$$

and inserting the expression for $\varphi^{*}$ from (6) yields

$$
\begin{aligned}
& \lambda_{\theta}+\frac{\left(r_{E} \lambda_{r_{E}}\right)^{2}}{\lambda_{\theta}}<0 \\
& \Rightarrow \lambda_{\theta}(t)<0 \forall 0 \leq t<1
\end{aligned}
$$

Similarly,

$$
\therefore \frac{\partial^{2} \mathscr{H}}{\partial \psi^{2}}=-\lambda_{r_{O}} \alpha \cos \psi-\frac{\lambda_{\theta} \alpha \sin \psi}{r_{O}}>0
$$

and inserting the expression for $\psi^{*}$ from (7) yields

$$
\begin{aligned}
& \lambda_{\theta}+\frac{\left(r_{O} \lambda_{r_{O}}\right)^{2}}{\lambda_{\theta}}<0 \\
& \Rightarrow \lambda_{\theta}(t)<0 \forall 0 \leq t<1
\end{aligned}
$$

The expressions for $\varphi^{*}$ and $\psi^{*}$ given in Equations (6) and (7) can be used to rewrite the state and co-state equations only in terms of the states and co-states. A nonlinear TwoPoint Boundary Value Problem (TPBVP) on the interval $t=$ $[0, \mathrm{~T}]$ is obtained:

$$
\begin{aligned}
\dot{r}_{E}= & \frac{\lambda_{r_{E}} r_{E}}{\sqrt{\lambda_{r_{E}}^{2} r_{E}^{2}+\lambda_{\theta}^{2}}}, r_{E}(0)=r_{E_{0}} \\
\dot{r}_{O}= & \frac{-\alpha \lambda_{r_{O}} r_{O}}{\sqrt{\lambda_{r_{O}}^{2} r_{O}^{2}+\lambda_{\theta}^{2}}}, r_{O}(0)=r_{O_{O}} \\
\dot{\theta}= & \frac{\lambda_{\theta}}{r_{E} \sqrt{\lambda_{r_{E}}^{2} r_{E}^{2}+\lambda_{\theta}^{2}}} \\
& -\frac{\alpha \lambda_{\theta}}{r_{O} \sqrt{\lambda_{r_{O}}^{2} r_{O}^{2}+\lambda_{\theta}^{2}}}, \theta(0)=\theta_{0} \\
\dot{\lambda}_{r_{E}}= & 4 r_{E}-2 r_{O} \cos \theta \\
& +\frac{\lambda_{\theta}^{2}}{r_{E}^{2} \sqrt{\lambda_{r_{E}}^{2} r_{E}^{2}+\lambda_{\theta}^{2}}}, \lambda_{r_{E}}(T)=0 \\
\dot{\lambda}_{r_{O}}= & 2 r_{O}-2 r_{E} \cos \theta \\
& -\frac{\alpha \lambda_{\theta}^{2}}{r_{O}^{2} \sqrt{\lambda_{r_{O}}^{2} r_{O}^{2}+\lambda_{\theta}^{2}}}, \lambda_{r_{O}}(T)=0 \\
\dot{\lambda}_{\theta}= & 2 r_{E} r_{O} \sin \theta, \lambda_{\theta}(T)=0,0 \leq t \leq T
\end{aligned}
$$

\section{A. The End-Game}

...Isaac's method entails the retrograde integration of eqs. (10), which obviates the need to solve the TPBVP. However, the controls do not feature in the integrant of the cost functional (2) and at time $\mathrm{t}=\mathrm{T}$ the co-states vanish. It 
is therefore impossible at time $\mathrm{T}$ to calculate the "initial" (terminal) controls by applying the PMP and maximizing and minimizing the Hamiltonian (4); obviously, equations (10) don't apply at time T because $\sqrt{\lambda_{r_{E}}^{2} r_{E}^{2}+\lambda_{\theta}^{2}}=0$. This is an interesting new wrinkle. The end game requires special attention.

Since the end state is free - that's why the co-states vanish at $\mathrm{t}=\mathrm{T}-$ the rElay's and rOver's optimal strategies at $\mathrm{t}=\mathrm{T}$ are myopic: according to the PMP, and also from first principles, the rElay would want the integrand in the cost functional

$$
L\left(r_{E}, r_{O}, \theta\right)=2 r_{E}^{2}+r_{O}^{2}-2 r_{E} r_{O} \cos \theta
$$

to be minimized. Similarly, the rOver would want the integrand in the cost functional to be maximized. Now, since the control variables $\varphi$ and $\psi$ do not directly feature in L, the rElay minimizes and the rOver maximizes the temporal derivative of $\mathrm{L}$, evaluated at $\mathrm{t}=\mathrm{T}$ :

$$
\begin{aligned}
\frac{d}{d t} L= & 2\left[2 r_{E} \cos \varphi+\alpha r_{O} \cos \psi-r_{O} \cos \varphi \cos \theta-\right. \\
& \left.\alpha r_{E} \cos \psi \cos \theta+r_{E} r_{O} \sin \theta\left(\alpha \frac{1}{r_{O}} \sin \psi-\frac{1}{r_{E}} \sin \varphi\right)\right] \\
= & 2\left\{\left(2 r_{E}-r_{O} \cos \theta\right) \cos \varphi-r_{O} \sin \theta \sin \varphi\right. \\
& \left.+\alpha\left[\left(r_{O}-r_{E} \cos \theta\right) \cos \psi+r_{E} \sin \theta \sin \psi\right]\right\}
\end{aligned}
$$

The rElay and rOver solve the respective static optimizations

$$
\min _{\varphi}\left[\left(2 r_{E}-r_{O} \cos \theta\right) \cos \varphi-r_{O} \sin \theta \sin \varphi\right]
$$

and

$$
\max _{\psi}\left[\left(r_{O}-r_{E} \cos \theta\right) \cos \psi+r_{E} \sin \theta \sin \psi\right]
$$

Concerning eq. (11): minimizing in $\varphi$ the derivative of the integrand at time $\mathrm{t}=\mathrm{T}$ yields the rElay's optimal terminal control

$$
\begin{aligned}
& \cos \left(\varphi^{*}(T)\right)=\frac{r_{O_{T}} \cos \theta_{T}-2 r_{E_{T}}}{\sqrt{4{r_{E_{T}}}^{2}+r_{O_{T}}{ }^{2}-4 r_{E_{T}} r_{O_{T}} \cos \theta_{T}}}, \\
& \sin \left(\varphi^{*}(T)\right)=\frac{r_{O_{T}} \sin \theta_{T}}{\sqrt{4{r_{E_{T}}}^{2}+r_{O_{T}}{ }^{2}-4 r_{E_{T}} r_{O_{T}} \cos \theta_{T}}},
\end{aligned}
$$

provided that $r_{E_{T}}, r_{O_{T}}$ and $\theta_{T}$ are s.t. the following is not the case: $r_{E_{T}}=\frac{1}{2} r_{O_{T}}$ and $\theta_{T}=0$. Here, $\left(r_{E_{T}}, r_{O_{T}}, \theta_{T}\right)$ denote the state at the terminal time $\mathrm{t}=\mathrm{T}$.

When $\theta_{T}=0$, eq. (11) yields

$$
\varphi^{*}(T)= \begin{cases}0 & \text { if } r_{E_{T}}<\frac{1}{2} \mathrm{r}_{O_{T}} \\ \pi & \text { if } \quad r_{E_{T}}>\frac{1}{2} \mathrm{r}_{O_{T}}\end{cases}
$$

An inspection of Fig. 1 tells us that in the end game, at time $\mathrm{t}=\mathrm{T}$, the rElay heads toward the midpoint $\mathrm{M}$ of the segment $\overline{B O}$. This, provided $\mathrm{E}$ is not already at the midpoint M.
Concerning eq. (12): maximizing in $\psi$ the derivative of the integrand at time $\mathrm{t}=\mathrm{T}$ yields

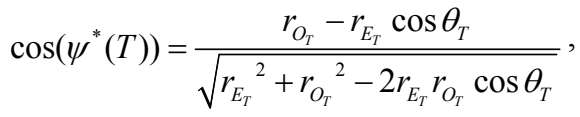

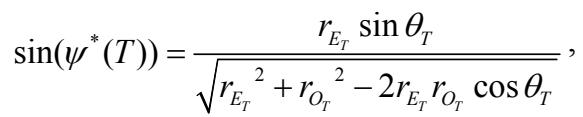

provided that $r_{E_{T}}, r_{O_{T}}$ and $\theta_{T}$ are s.t. the following is not the case: $r_{O_{T}}=r_{E_{T}}$ and $\theta_{T}=0$.

When $\theta_{T}=0$, eq. (12) yields

$$
\psi^{*}(T)=\left\{\begin{array}{lll}
0 & \text { if } & \mathrm{r}_{O_{T}}>r_{E_{T}} \\
\pi & \text { if } & \mathrm{r}_{O_{T}}<r_{E_{T}}
\end{array}\right.
$$

An inspection of Fig. 1 tells us that in the end game, at time $\mathrm{t}=\mathrm{T}$, the rOver runs away from the rElay. The players'

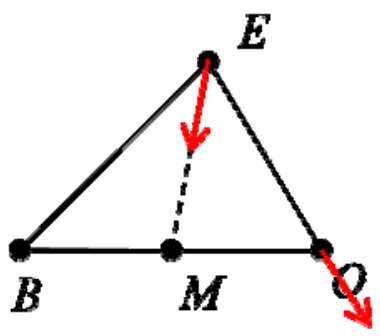

Fig. 2. The end game

strategies in the end game are shown in Figure 2.

\section{END GAME IN DIFFERENTIAL GAME}

The end game strategies shown in Fig. 2 don't apply when $r_{E}=\frac{1}{2} r_{O}$ and $\theta=0$, namely, they don't apply at point Mreferred to as the "sweet spot"; we cannot start the backward integration at $\mathrm{M}$.

In the differential game there is a line of "sweet spots", $\left(\frac{1}{2} r_{o_{T}}, r_{o_{T}}, 0\right)$, which is parameterized by $r_{o_{T}}, r_{o_{T}}>0$. Each

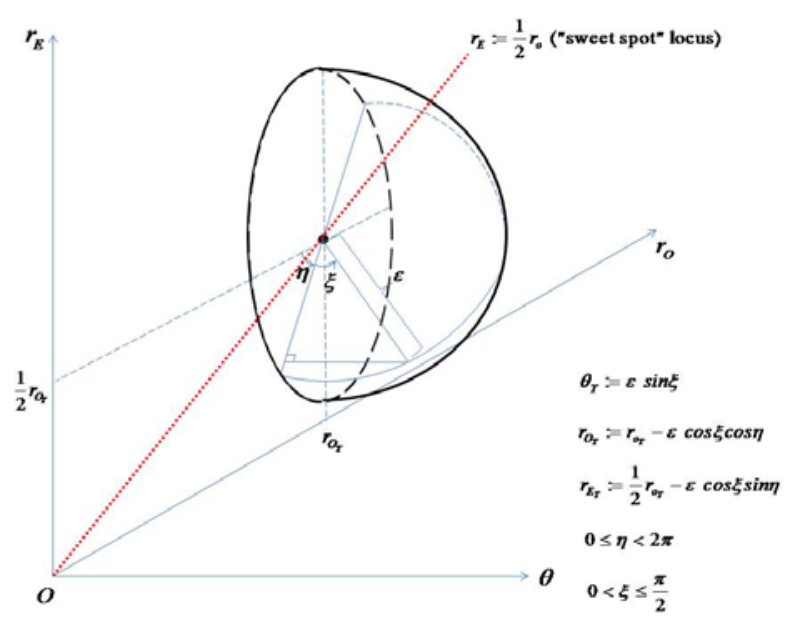

Fig. 3. End State's Manifold 
"sweet spot" on the said (red) line is encapsulated in a small hemispherical terminal manifold, as shown in Fig.3

We back off from "sweet spots" and the retrograde integration of the characteristics equations is initiated from end states $\left(r_{E_{T}}, r_{o_{T}}, \theta_{T}\right)$ on a hemispherical manifold, as shown in Fig. 3, where

$$
\begin{gathered}
\theta_{T}=\varepsilon \sin \xi \\
r_{O_{T}}:=r_{o_{T}}-\varepsilon \cos \xi \cos \eta \\
r_{E_{T}}:=\frac{1}{2} r_{o_{T}}-\varepsilon \cos \xi \sin \eta \\
0 \leq \eta<2 \pi
\end{gathered}
$$

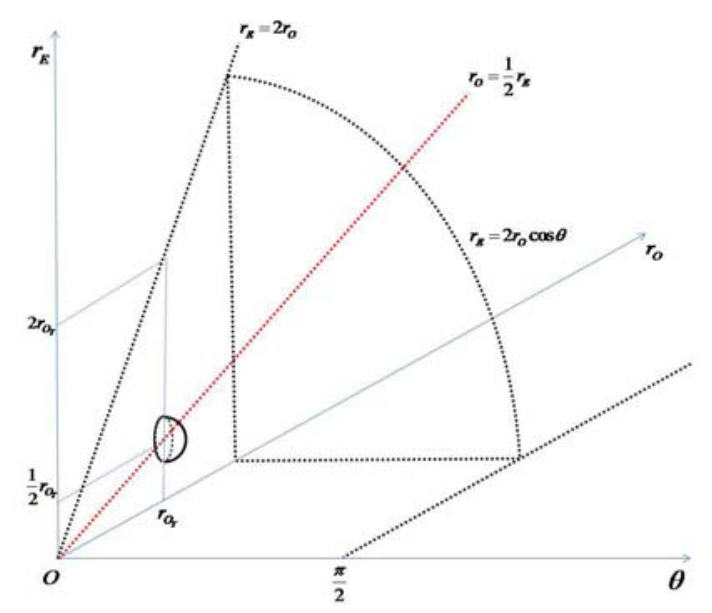

Fig. 4. State Space

$$
0<\xi \leq \frac{\pi}{2}
$$

The end states associated with the "sweet spot" $\left(\frac{1}{2} r_{o_{T}}, r_{o_{T}}, 0\right)$ reside on a small hemispherical terminal manifold, as shown in Fig. 4, where the state space of the differential game is illustrated.

Without loss of generality we confine our attention to $r_{o_{T}}=1$. The family of optimal trajectories is thus parameterized by $0<\xi \leq \frac{\pi}{2}$ and $0 \leq \eta<2 \pi$. The retrograde integration stops when the boundary of the state space is reached and $r_{E}=2 r_{o} \cos \theta$.

The optimal control of the rElay at $\mathrm{t}=\mathrm{T}$ is given by the solution of the end game where the end state on the hemispherical terminal manifold is $r_{E_{T}}, r_{o_{T}}, \theta_{T}$. It is

$$
\begin{aligned}
& \cos \left(\varphi^{*}(T)\right)=\frac{r_{O_{T}} \cos \theta_{T}-2 r_{E_{T}}}{\sqrt{4{r_{E_{T}}}^{2}+r_{O_{T}}{ }^{2}-4 r_{E_{T}} r_{O_{T}} \cos \theta_{T}}}, \\
& \sin \left(\varphi^{*}(T)\right)=\frac{r_{O_{T}} \sin \theta_{T}}{\sqrt{4{r_{E_{T}}}^{2}+r_{O_{T}}{ }^{2}-4 r_{E_{T}} r_{O_{T}} \cos \theta_{T}}},
\end{aligned}
$$

Similarly, the optimal control of the rOver at $\mathrm{t}=\mathrm{T}$ given by the solution of the end game is

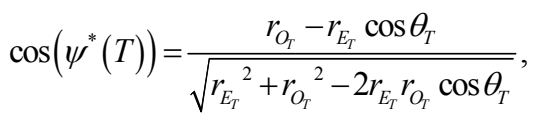

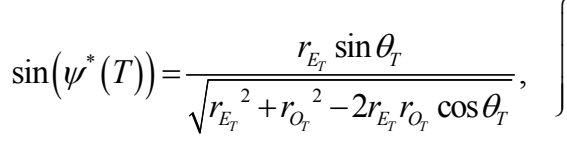

provided that the end state is not $r_{E_{T}}=r_{O_{T}}, \theta_{T}=0$; when this is the case, from first principles we conclude that the rOver's control $\psi^{*}(T)=0$. Thus, having backed off from the "sweet spot", one considers the terminal states on the small hemisphere which encapsulates the "sweet spot" shown in Fig. 3. The end states on the small hemisphere around the "sweet spot" are parameterized as follows.

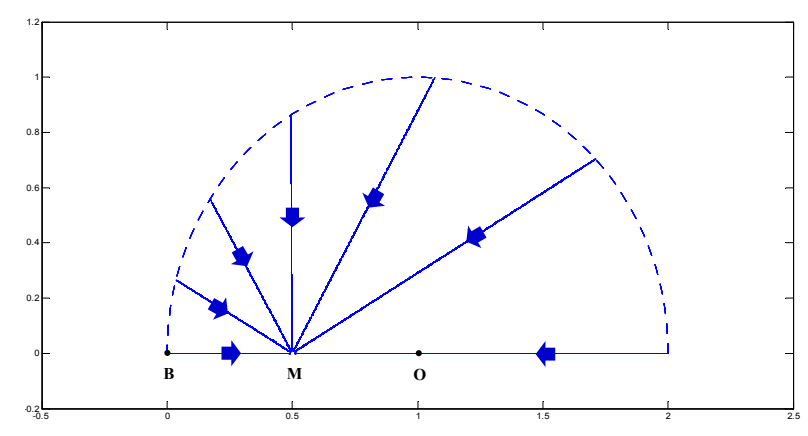

Fig. 5. The optimal flow field when the rOver is stationary

$$
\begin{aligned}
& \theta_{T}:=\varepsilon \sin \xi \\
& r_{O_{T}}:=r_{o_{T}}-\varepsilon \cos \xi \cos \eta \\
& r_{E_{T}}:=\frac{1}{2} r_{o_{T}}-\varepsilon \cos \xi \sin \eta \\
& 0 \leq \eta<2 \pi \\
& 0 \leq \xi<\frac{\pi}{2} \\
& 0<\varepsilon \ll 1, \text { fixed }
\end{aligned}
$$

The optimal controls at $\mathrm{t}=\mathrm{T}$ for end states on the hemispherical terminal manifold are obtained by inserting eqs. (15) into the terminal control eqs. (13) and (14). After that first step, one integrates eqs. (10) in retrograde fashion.

The complete solution of the one sided, simpler, optimal control problem when the rOver is stationary is shown in Fig. 5. Note the small semicircular terminal manifold centered at the "sweet spot" $M$. 


\section{NUMERICAL RESULTS}

Guided by the suboptimal solution and the solution of the one - sided optimal control problem, the differential game is solved using Isaacs method [2], namely, the retrograde integration of the characteristics' equations (10), as discussed in section II and III. In the figures below, the spatial results are shown. The following numerical results show the solution of the differential game where $T=0.25, \alpha=1, r_{E_{0}}=.5, r_{O_{0}}=1$ and $\theta_{0}=\frac{\pi}{6}$.

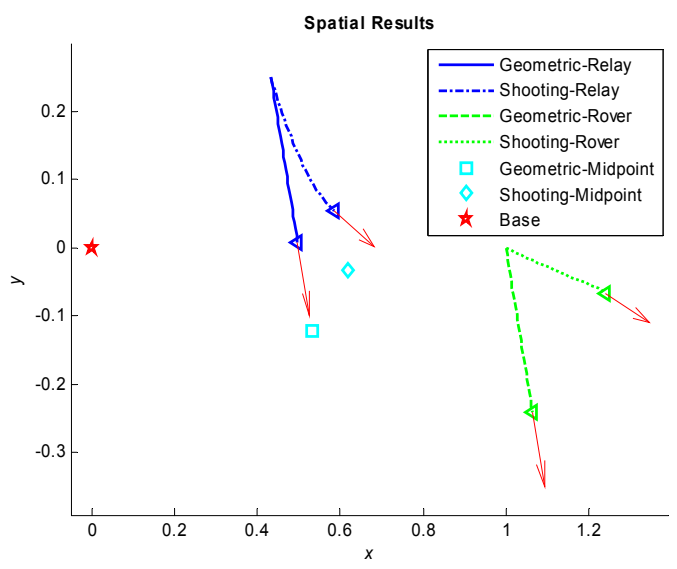

Fig. 6. Spatial Results for

$$
\boldsymbol{T}=.25, \alpha=1, \boldsymbol{r}_{E_{0}}=.5, \boldsymbol{r}_{\boldsymbol{O}_{0}}=1 \text { and } \boldsymbol{\theta}_{0}=\frac{\pi}{6}
$$

The following numerical results show the solution of the min-max problem where $T=.49, \alpha=1, r_{O_{0}}=1$ and $\theta_{0}=\frac{\pi}{3}$.

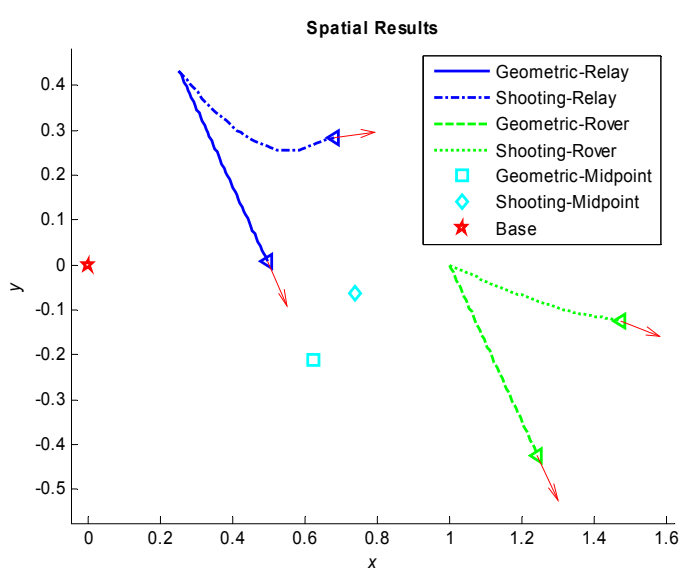

Fig. 7. Spatial Results for

$$
\boldsymbol{T}=.49, \boldsymbol{\alpha}=1, \boldsymbol{r}_{\boldsymbol{E}_{0}}=.5, \boldsymbol{r}_{\boldsymbol{O}_{0}}=1 \text { and } \boldsymbol{\theta}_{0}=\frac{\pi}{3}
$$

Optimal trajectories for additional scenarios are illustrated in Figures 8 and 9. The point is that although initially the relay, rover and base are collinear and the rover is between the relay and the base, the engagement is not linear. The rover breaks out, the relay goes around, and the optimal trajectories are curved.

\section{CONCLUSIONS}

This paper develops optimal guidance laws for a Relay MAV in support of extended range ISR. The algorithm is based upon the solution of a min-max optimization problem, namely, the solution of the differential game, which represents a worst case scenario. Heuristic Relay guidance strategies are also provided. These are derived using a geometry based (sub)optimality principle, and also the solution of the one-sided Relay optimal control problem, where the Rover is considered stationary. Both the heuristic method and the solution of the one-sided optimal control problem shown in Fig. 5 provide corroborating results which were then employed to gain insight into the solution of the differential game. The optimal control and differential game's solution exhibits interesting behavior: the optimal flow field namely the optimal trajectories will converge to the family of end states $r_{E}=\frac{1}{2} r_{O}, \theta=0$. The latter is a singular trajectory, provided the speed ratio $\alpha \leq 2$.

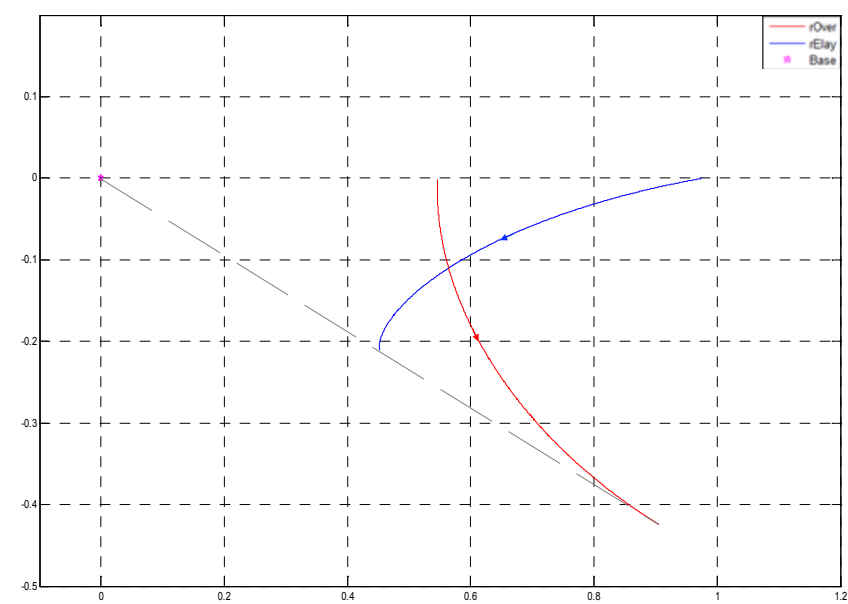

Fig. 8. Optimal trajectories of Rover and Relay.

$$
\alpha=1, \xi=150^{\circ}, r_{O_{T}}=1
$$

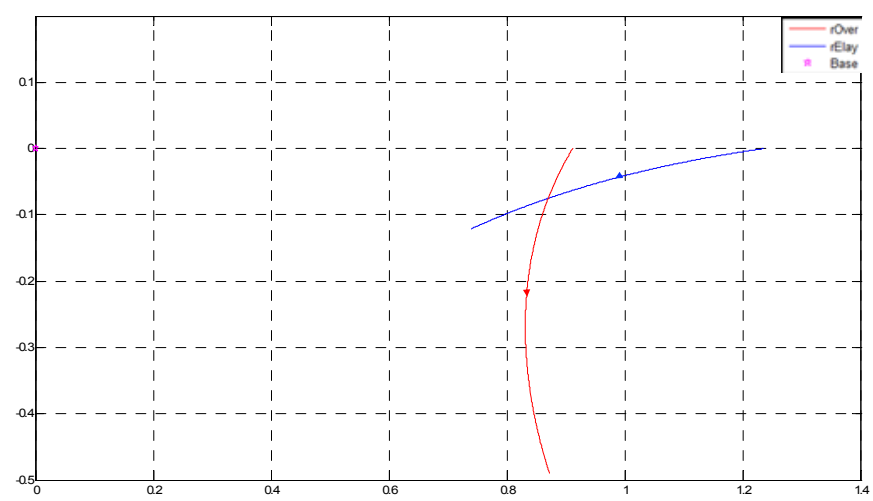

Fig. 9. Optimal Trajectories of Rover and Relay When

$$
r_{E_{T}}=\frac{3}{4}, r_{O_{T}}=1, \alpha=1, \theta_{T}=20^{\circ}
$$




\section{APPENDIX}

Geometric solution of the one-sided optimal control problem

The optimal strategy of the rElay is to point toward the midpoint $\mathrm{M}$ of the Base-rOver segment. This can be deduced from the following elementary Euclidean geometry result.

It is well known that the locus of all points such that the sum of the distances from two fixed points is constant, is an ellipse. Thus, the following is of some interest [4].

Theorem 1 The Locus of all points such that the sum of the squares of the distances from two fixed points is constant, is a circle centered at the midpoint of the segment formed by the two fixed points. The radius of this circle is

$$
R=\sqrt{d^{2}-f^{2}}
$$

where the sum of the squares of the distances is $2 d^{2}$ and the distance between the fixed points is $2 f$; obviously, $d \geq f$.

\section{Proof:}

Let the fixed points $F_{1}$ and $F_{2}$ be on the $x$-axis $\left(F_{1}=(f, 0)\right.$, $\left.F_{2}=(-f, 0)\right)$ as shown in the figure below.

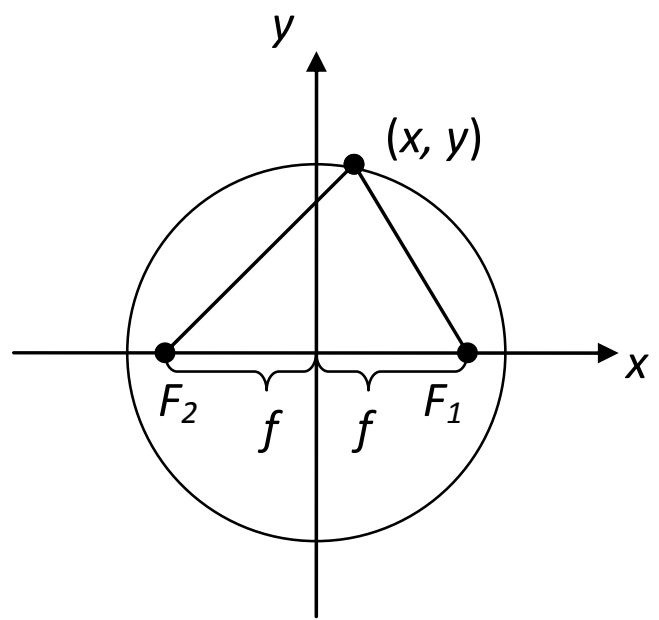

Fig. 10. Schematic of Fixed Points Showing Isocost Circle

The sum of the squares of the distances is calculated as

$$
\begin{aligned}
2 d^{2} & =(f+x)^{2}+y^{2}+(f-x)^{2}+y^{2} \\
& =2 f^{2}+2 x^{2}+2 y^{2} \\
\Rightarrow & x^{2}+y^{2}=d^{2}-f^{2}
\end{aligned}
$$

This is the equation of a circle centered at the origin, whose radius is

$$
R=\sqrt{d^{2}-f^{2}}
$$

This result appeared in [5].

Remark: The loci of constant costs, $2 d^{2}$, are concentric circles where the minimum cost is found at the midpoint of the line formed by $F_{1}$ and $F_{2}$, where $d=f$.

Extension: The Locus of all points such that the weighted sum of the squares of the distances from two fixed points is constant, is a circle centered on the segment formed by the two fixed points and is at a distance of $(1-2 \alpha) f$ from this segment's midpoint. The radius of this circle is

$$
R=\sqrt{d^{2}-4 \alpha(1-\alpha) f^{2}}
$$

where $d^{2}$ is the specified weighted sum of the squares of the distances, the distance between the fixed points is $2 f$; and the weight is $\alpha$; if $\alpha<0$ or $\alpha>1$ this is true $\forall d>0$, and if $0 \leq \alpha \leq 1, d>2 f \sqrt{\alpha(1-\alpha)}$. Note: When the weight $\alpha=1 / 2$, need $d>f$.

Proof: The weighted sum of the squares of the distances is calculated as

$$
\begin{aligned}
d^{2}= & \alpha\left[(f+x)^{2}+y^{2}\right]+(1-\alpha)\left[(f-x)^{2}+y^{2}\right] \\
& =\alpha f^{2}+\alpha x^{2}+2 \alpha f x+\alpha y^{2}+(1-\alpha) f^{2} \\
& +(1-\alpha) x^{2}-2(1-\alpha) f x+(1-\alpha) y^{2} \\
& =f^{2}+x^{2}+y^{2}-2 f x(1-2 \alpha) \\
& =[x-(1-2 \alpha) f]^{2}+f^{2}+y^{2}-(1-2 \alpha)^{2} f^{2} . \\
\Rightarrow & {[x-(1-2 \alpha) f]^{2}+y^{2}=d^{2}-4 \alpha(1-\alpha) f^{2} }
\end{aligned}
$$

The circular shape of the isocost surfaces is conducive to the rElay heading toward the center, namely, point $\mathrm{M}$.

\section{REFERENCES}

[1] Goldenberg, D., Lin, J., Morse, A. S., Rosen, B. E., and Yang, Y. R., "Towards Mobility as a Network Control Primitive," Mobihoc '04, ACM, 24-26 May 2004

[2] Isaacs, R.: Differential Games - a mathematical theory with application to warfare and pursuit, control and optimization. SIAM 1965

[3] L. S. Pontryagin, V. G. Boltyanskii, R. V. Gamkrelidze, and E. F. Mishchenko, The Mathematical Theory of Optimal Processes, John Wiley, 1962

[4] V. Gutenmacher and N. B. Vasilyev, Lines and Curves, Birkhäuser Boston, 2004, pp. 23-24 